release assays (IGRA)) have been useful in detecting $\mathrm{Mtb}$ infection in exposed individuals. We assessed infections in TB cases and their exposed household contacts (HHC) using an in-house optimised IGRA, the QuantiFERON-TB Gold in Tube (QFT-GIT) and QFT-Plus (QFT+).

Methods For the in-house IGRA, we analysed 266 active TB patients and 759 HHC (256 tuberculin skin test-positive and 503 test-negative, TST + and TST- respectively) at baseline and 6 months. In a separate study we assessed QFT-GIT and QFT-plus responses using samples from $72 \mathrm{~TB}$ cases and 69 HHC at baseline. QFT-GIT has 3 Mtb-specific antigens: ESAT6, CFP10 and TB7.7 while QFT-plus has long and short peptides of ESAT-6 and CFP-10, designed to induce CD4+ and $\mathrm{CD} 8+\mathrm{T}$ cell responses respectively.

Results IFN- $\gamma$ responses were lowest in TST- compared to both TST + and TB patients at baseline $(\mathrm{p}<0.0001$ for both), with $32 \%$ IGRA-positive compared to $76 \%$ and $73 \%$, respectively using in-house IGRA. HHC sleeping in the same room with TB patient had a significantly higher IGRA conversion rate by 6 months compared to those sleeping further away $(p=0.0004)$. We also observed a significant decline in IGRA IFN- $\gamma$ levels by 6 months of TB treatment $(\mathrm{p}<0.0001)$. Among QFT-positive TB patients, smear-positive was $57 \%$ and culture-positive was $62 \%$. The IFN- $\gamma$ concentration between TB1 and TB 2 was similar, while, QFT-GIT had a significantly higher response than TB 1 and TB 2 for both TB patients $(\mathrm{p}=0.003)$ and HHC $(\mathrm{p}=0.0005)$.

Conclusion Our findings show that IGRA conversion is significantly increased in HHC with highest exposure but that IGRA -positive cannot predict risk of progression to active TB. We also found that QFT-GIT was quantifiably better than QTFplus in our setting, limiting the 'grey zone' of indeterminate results.

\section{PO 8490 PROMOTING GOOD DATA MANAGEMENT PRACTICES IN CLINICAL RESEARCH IN RESOURCE-POOR SETTINGS}

Harry Van Loen*, Diana Arango, Hanne Landuyt, Christophe Burm, Yven Van Herrewege. Institute of Tropical Medicine, Antwerp, Belgium

\subsection{6/bmjgh-2019-EDC.118}

Background Accurate and timely data management (DM) is of key importance in clinical research to generate high-quality and GCP-compliant data for analysis and/or sharing. Our objective is to strengthen the capacity for DM in clinical research in resource-poor settings by organising several teaching initiatives.

Methods Our teaching initiatives have a twofold approach. First, a generic and comprehensive approach with capacity building on various thematical modules. These include a research component (overviewing the research data management procedures) and a technological component (introducing databases and software). In addition, a component on legislation, guidelines and standards specific towards DM is discussed, as well as a project management component on how to organise DM efficiently and timely. Second, we apply a more focused and study-specific approach which details roles and responsibilities in data management, milestones and documentation practices. Both approaches are based upon successful implementation in EDCTP-funded clinical trials, such as the $4 \mathrm{ABC}$, PREGACT and Microbicide Safety Biomarkers studies, as well as the FP7 sponsored NIDIAG project. The target audience comprises various study stakeholders such as data managers, IT administrators, clinicians, laboratory researchers and statisticians, coming from sub-Saharan Africa, South-East Asia and Latin America.

Results A teaching model for promoting Good Data Management Practices has been developed with theory- and practicebased modules. This model is used at face-to-face workshops in remote settings and has been re-used by colleagues and implemented by other research institutions to promote further capacity building and sustainable development in the South. In addition, it has led to mutual learning and enhanced institutional and personal North-South collaborations.

Conclusion There is a clear case for promoting DM and providing guidelines for Good Data Management Practices. Our twofold approach has enabled the successful conduct of GCP compliant non-commercial clinical trials in the South.

\section{PO 8492 REPEATED ARTEMISININ-BASED TREATMENT ON MALARIA SEXUAL PARASITE DISTRIBUTION IN POPULATION LIVING IN A MALARIA-ENDEMIC AREA OF BURKINA FASO}

Amidou Diarra*, Issiaka Soulama, Issa Nebie, Maurice Ouattara, Moise Kabore, Benjain Sombie, Alfred B Tiono, Alphonse Ouedraogo, Sodiomon B Sirima. Centre national de recherche et de formation sur le paludisme, Ouagadougou, Burkina Faso

\subsection{6/bmjgh-2019-EDC.119}

Background Malaria elimination and its ultimate eradication will require drugs targeting all stages of the parasite's life cycle. Yet, very few drugs are known to be effective on the sexual stages (gametocytes) of Plasmodium falciparum. Artemisinin-based combination therapy (ACT) has been shown to have some early-stage gametocytocidal effects on in vitro and in feeding experiments. However, field studies showed that artesunate reduces but does not prevent post-treatment transmission of $P$. falciparum to mosquitos.

Methods 763 children and adult patients with acute uncomplicated Plasmodium sp. malaria were included in a phase IIIb/IV comparative, randomised, multi-centre, open label, parallel 3arm clinical trial to assess safety and efficacy of repeated administration of pyronaridine-artesunate, dihydroartemisininpiperaquine or artemether-lumefantrine or artesunate-amodiaquine over a two-year period. Drugs were given based on the body weight and volunteers were followed up for 42 days. Clinical signs and symptoms were recorded and filter paper and blood smears collected during each visit. Malaria parasites were assessed and parasite density development stages determined by light microscopy.

Results $P$. falciparum gametocyte was $1.9 \%$, during the two years of follow-up. From the three treatment arms, artesunateamodiaquine was the arm bearing more $P$. falciparum gametocyte with $68.7 \%$, dihydroartemisinin-piperaquine accounted for $6.3 \%$ and pyronaridine-artesunate for 25\%. P. falciparum gametocyte was more pronounced in populations having parasite density $\leq 100000$ parasites $/ \mu \mathrm{l}$ compared to above parasitaemia.

Conclusion Repeated ACTs treatment didn't clear P. falciparum gametocyte in a population infected with uncomplicated malaria. 